\title{
Ausnahmebestimmung für Verchromen in der Schweiz
}

\author{
Die Harmonisierung des Schweizer Chemikalienrechts mit der EU hat zu einem Anwendungs-Verbot \\ für Chromsäure geführt. Die Arbeitsgruppe REACH der Schweizer Stiftung für Oberflächentechnik \\ (SSO) hat sich dafür eingesetzt, für die Schweiz hier eine "pragmatische“ Lösung zu finden. Anders als \\ in der EU gilt nun in der Schweiz eine Ausnahmebestimmung für das Hart- und Glanzverchromen.
}

Bereits seit dem 1. Februar 2017 ist das Inverkehrbringen und die berufliche und gewerbliche Verwendung der wichtigsten sechswertigen Chrom-Verbindungen in der Schweiz grundsätzlich verboten. Die Schweiz hat damit eine Änderung des Chemikalienrechts übernommen, die in der EU schon seit einiger Zeit gilt. Die EU geht mit ihrem Chemikalienrecht grundsätzlich so vor, dass besonders giftige oder besorgniserregende Stoffe verboten werden. Wer ein besonderes Interesse geltend machen und beweisen kann, dass er ein Maximum an Schutzmaßnahmen anwenden kann, kann bei der zuständigen EUBehörde eine sogenannte Autorisierung, das heißt eine Ausnahmebewilligung, für die Verwendung des Stoffes beantragen. Mit diesem Vorgehen kann erreicht werden, dass wichtige industrielle Prozesse und Verwendungen mit dem kritischen Stoff weiterhin möglich bleiben.

\section{Keine eigene Stoffbeurteillung}

Der Bundesrat hat beschlossen, das Schweizer Chemikalienrecht mit dem europäischen zu harmonisieren und keine „eigenen“ Stoffbeurteilungen vorzunehmen. Das scheint sinnvoll. Nur: Die Prozedur der Bewilligungserteilung in der EU dauert etwa eineinhalb Jahre und die nötigen Nachweise und Belege erfordern Studien und Abklärungen, die leicht um die 100.000 Euro kosten. Zudem können Schweizer Firmen keine Anträge bei der zuständigen EU-Behörde einreichen, da die Schweiz nicht Mitglied der EU ist.

Nach Auffassung der Arbeitsgruppe REACH der SSO ist das EU Chemikalienrecht nicht gerade das, was Schweizer KMU vom Regulator erwarten. Wegen der großen damit verbundenen Probleme ist diese EU-Regelung auch schon als das „Ende des Verchromens in Europa“ bezeichnet worden. Hinzu kommt die Wettbewerbsverzerrung, wenn der Schweizer Betrieb darauf angewiesen ist, dass ein europäischer Konkurrent ein Gesuch einreicht, bevor er selber weiterproduzieren darf.

\section{Problem für Verchromer stark entschärft}

Das Problem wurde nun für die Schweizer Verchromer stark entschärft. Anders als in der EU ist in der Schweizerischen Verordnung eine Ausnahmebestimmung aufgenommen worden für Verwendungen in Prozessen, in deren Endprodukten Chrom nicht in sechswertiger Form vorliegt. Damit sind Betriebe, die verchromen, in deren Produkten aber kein giftiges Chromat, sondern nur ungiftiges Chrom vorhanden ist, vom Chrom(VI)-Verbot ausgenommen und können wie bisher legal weiterarbeiten.

Die verchromenden Betriebe müssen im Gegenzug dazu bereit sein, einen neuen Grenzwert in Bezug auf die Chrom(VI)Exposition der Mitarbeitenden am Arbeitsplatz umzusetzen. Mit diesem Wert soll sichergestellt werden, dass das Schutzniveau der Mitarbeitenden in Schweizer verchromenden Betrieben mindestens dem Schutzniveau in den sogenannten „Autorisierten Betrieben“ in der EU entspricht. Diese neue Vorgabe ist derzeit bei den Schweizer Bundesbehörden in Ausarbeitung.

Nach Meinung der SSO macht diese Lösung Sinn, da die Gefahren durch Chromat nur am Arbeitsplatz bestehen. Das verchromte Produkt enthält kein Chromat und für den Kunden beziehungsweise Konsumenten besteht deshalb keine Gefahr.

Am Arbeitsplatz hat aber die Schweiz aus Sicht der SSO mit der Tätigkeit der Schweizerischen Unfallversicherung und der Arbeitsinspektorate eine alte und gut funktionierende Tradition des Arbeitnehmerschutzes. Für jene Betriebe, die den neuen Chromat-Expositionsgrenzwert am Arbeitsplatz einhalten ist es laut SSO absolut gerechtfertigt, das komplizierte, langsame und teure Autorisierungsverfahren der EU zu umgehen. //

\section{Kontakt}

SSO Sekretariat Arbeitsgruppe REACH

Dr. Jürg Liechti, Gerlafingen

Tel. +41326744525

juerg.liechti@neosys.ch 